\title{
Efficient Synthesis of Azido Sugars using Fluorosulfuryl Azide Diazotransfer Reagent
}

\author{
Joshua M. Kofsky, ${ }^{[a]}$ Gour C. Daskhan,${ }^{[b]}$ Matthew S. Macauley, ${ }^{[b, c]}$ and Chantelle J. Capicciotti ${ }^{* a, d]}$
}

[a] Department of Chemistry, Queen's University, Kingston, K7L 3N6, Canada [b] Department of Chemistry, University of Alberta, Edmonton, T6G 2G2, Canada [c] Department of Medical Microbiology and Immunology,

University of Alberta, Edmonton, T6G 2E1, Canada [d] Department of Biomedical and Molecular Sciences; Department of Surgery, Queen's University, Kingston, K7L 3N6, Canada

*Correspondence to: c.capicciotti@queensu.ca

\begin{abstract}
Azide-containing sugars are important tools for the synthesis of biologically relevant 1,2-cis-glycosides and for bioconjugation chemistry. Previous strategies for the installation of a non-participating $\mathrm{C} 2$-azido functionality use harsh conditions and long reaction times. Herein, we report the synthesis of azido sugars using fluorosulfuryl azide $\left(\mathrm{FSO}_{2} \mathrm{~N}_{3} ; 1\right)$ with a $\mathrm{Cu}$ (II) catalyst as a safe and efficient diazotransfer reagent. Common hexosamine substrates were converted to 2-azido-2-deoxy sugars in less than 5 minutes in quantitative yield. Glycosyl donors with orthogonal protecting groups were readily prepared from these azido sugars with good overall yield and a single column purification. The diazotransfer protocol was also efficiently used on other amino sugar derivatives, including aminoglycosides and substrates with amine-containing linkers. This optimized method will expand access to important non-participating C2-azido protecting groups and other azido sugar derivatives.
\end{abstract}

\section{Introduction}

Azide-containing carbohydrates are important and versatile tools in synthetic chemistry and chemical biology. They are widely used in the synthesis of amino sugars, glycopeptides and multivalent glycoconjugates, and for biorthogonal labelling strategies to study cellular glycans. ${ }^{[1]}$ Glycosides containing 2-amino-2-deoxysugars are present in glycoproteins, glycolipids, glycosaminoglycans, and blood group oligosaccharides where they play important biological roles in protein recognition by lectins, antibodies, and enzymes ${ }^{[2]}$ Many of these biologically relevant amino sugars contain 2- $\mathrm{N}$-acetamido-2-deoxy-glucosides and -galactosides, which are connected to other sugar residues through 1,2-cis or 1,2-trans glycosidic linkages. ${ }^{[2 \mathrm{~b}, 2 \mathrm{~d}, 3]}$ While 
1,2-trans glycosides can be reliably prepared with a participating group protecting the C2-amine, 1,2-cis glycosides are more challenging to selectively prepare and require non-participating protecting groups at $\mathrm{C}-2$. As such, $\mathrm{C} 2$-azido sugars are widely used in carbohydrate synthesis when non-participating moieties are required. ${ }^{[36,4]}$ Given the importance of the C2-azido functionality in synthesis, and the prevalence of azido sugars used in biorthogonal chemistry and glycoconjugate preparation, simple and efficient strategies to prepare azido sugars are highly desirable.

Various synthetic strategies have been developed to prepare 2-azido-2-deoxysugars. Glycals are useful building blocks for installing a C2-azido moiety via ceric ammonium nitrate (CAN)promoted radical azidonitration in the presence of sodium azide, or azide ionic azidophenylselenylation protocols. ${ }^{[5]}$ Unfortunately, these conditions can suffer from poor stereoselectivity, longer reaction times and lower yields, particularly when starting from glucals, which often result in the formation of epimeric mixtures of 2-azido-2-deoxy-gluco and -manno pyranosides. ${ }^{[5,6]}$ More recent methods have since been developed starting from amino sugars that enable direct conversion to the azido derivatives while retaining stereochemistry through diazotransfer reactions. Diazotransfer on C2-amino sugars was first reported using trifluoromethanesulfonyl azide (triflyl azide; $\mathrm{TfN}_{3}$ ) as the diazotizing reagent under inert atmospheric conditions. ${ }^{[7]}$ Additional diazotransfer conditions and reagents have since been reported for the preparation of 2-azido-2-deoxysugars. ${ }^{[8]}$ Often these diazotransfer protocols require long reaction times, metal-based reagents, and can involve potentially hazardous (i.e. explosive and toxic) reagents or intermediates. ${ }^{[0]}$ For instance, the diazotransfer reagent imidazole-1-sulfonyl azide was once suggested to be a shelf-stable solid as the chloride salt, but later was found to be an explosion risk and prone to degradation to hydrazoic acid. ${ }^{[8 e, 10]}$ Thus, the development of efficient and safe diazotransfer protocols is critical for the use of azide protecting groups in oligosaccharide synthesis and the preparation of other azido sugars.

In 2019, Meng et al. reported the first use of fluorosulfuryl azide $\left(\mathrm{FSO}_{2} \mathrm{~N}_{3} ; \mathbf{1}\right)$ as a safe, efficient and highly reactive reagent for diazotransfer on organic amines under metal-free conditions. ${ }^{[11]}$ The $\mathrm{FSO}_{2} \mathrm{~N}_{3}$ (1) reagent could quantitatively convert primary amines to azides in 5 min and demonstrated a large substrate scope of $>40$ examples directly synthesized and $>1200$ examples assayed in a 96-well microtitre plate. The reagent is impact-resistant and showed little to no risk of acute oral toxicity, although it is friction-sensitive. ${ }^{[11]}$ This precludes many of the previously reported safety hazards associated with diazotransfer reagents, suggesting 1 as a suitably safe alternative diazotizing agent. Additionally, 1 can be efficiently prepared from a stable fluorosulfuryl imidazolium salt via sulfuryl fluoride exchange (SuFEx) click chemistry under an air atmosphere 
in 10 minutes (Scheme 1). ${ }^{[11-12]}$ While a large substrate scope was initially reported, ${ }^{[11]}$ diazotransfer using 1 with 2-amino-2-deoxysugar substrates to prepare orthogonally protected sugar building blocks has not been reported. We therefore sought to examine the use of diazotransfer reagent 1 on sugar substrates to determine if this reagent could improve the efficiency of synthesizing orthogonally protected azido sugars and azido sugar derivatives.

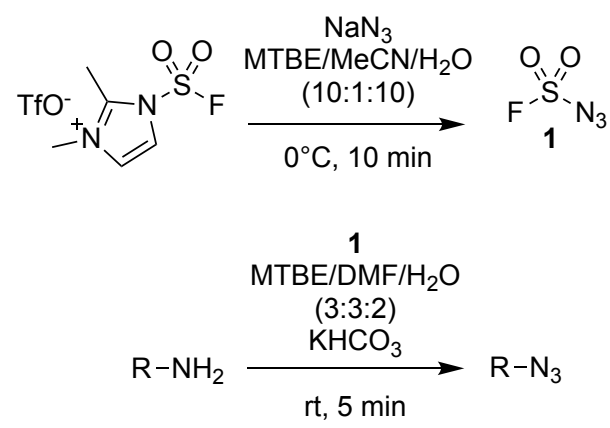

Scheme 1. Preparation of the diazotransfer reagent fluorosulfuryl azide $\left(\mathrm{FSO}_{2} \mathrm{~N}_{3} ; 1\right)$ and its use in the synthesis of organic azides from primary amines. ${ }^{[11]}$

\section{Results and Discussion}

We first prepared the diazotransfer reagent $\mathrm{FSO}_{2} \mathrm{~N}_{3}$ (1) in 10 min through sulfuryl fluoride exchange (SuFEx) in a biphasic mixture of water and methyl tert-butyl ether (MTBE) (Scheme 1). ${ }^{[11]} \mathrm{FSO}_{2} \mathrm{~N}_{3}$ (1) readily partitioned into the organic MTBE phase, and after confirming its formation by ${ }^{19} \mathrm{~F}-\mathrm{NMR}$ spectroscopy, this solution was directly used in diazotransfer reactions. Diazotransfer reactions with $\mathrm{FSO}_{2} \mathrm{~N}_{3}(1)$ were reported previously to have quantitative conversion of primary amines to the corresponding azides in $5 \mathrm{~min}$ at room temperature. ${ }^{[11]}$ Thus, we first attempted an equimolar reaction of 1 with galactosamine $\left(\mathrm{GaINH}_{2} ; \mathbf{2 a}\right)$, however after 5 min there was little conversion to the $\mathrm{GaIN}_{3}$ (3a) product. Increasing to two equivalents of 1 did increase the formation of the GalN $\mathrm{N}_{3}$ (3a) product, however only $54 \%$ conversion was observed (Fig. S1). While most amine substrates initially reported had quantitative conversion in 5 min to their respective azido-products, some sterically-crowded or electron-deficient amines had lower yields, even with longer reaction times and the use of excess equivalents of $\mathrm{FSO}_{2} \mathrm{~N}_{3}(1) \cdot{ }^{[11]}$ As complete conversion of the 2-amino-2-deoxysugar to the corresponding azide was not observed in $5 \mathrm{~min}$, we next sought to explore if we could optimize this diazotransfer reaction and maintain the fast reaction time for sugar substrates.

Metal catalysts, such as $\mathrm{CuSO}_{4}, \mathrm{ZnCl}_{4}$ or $\mathrm{NiCl}_{4}$, can reduce reaction times for diazotransfer on amino sugars using $\mathrm{TfN}_{3} .{ }^{[8 \mathrm{dd}]}$ While the diazotransfer reactions using $\mathrm{FSO}_{2} \mathrm{~N}_{3}(1)$ were reported to not benefit from metal catalyst addition, ${ }^{[11]}$ we sought to explore if these additives would improve the conversion efficiency of amino sugar substrates. We opted to use $\mathrm{CuSO}_{4}$ as this $\mathrm{Cu}^{\text {II-catalyst }}$ 
results in more efficient $\mathrm{TfN}_{3}$-mediated diazotransfer reactions and it can act as a colour indicator for reaction progress, with the solution changing from blue to green when the amine starting material is fully consumed. ${ }^{[8 a]}$ When equimolar $\mathrm{FSO}_{2} \mathrm{~N}_{3}(1)$ was used with catalytic $\mathrm{CuSO}_{4}(1.25$ mol\%), we did not observe full conversion in $5 \mathrm{~min}$ to the corresponding azido sugars when galactosamine $\left(\mathrm{GalNH}_{2} ; \mathbf{2 a}\right)$, glucosamine $\left(\mathrm{GlcNH}_{2} ; \mathbf{2 b}\right)$, or mannosamine $\left(\mathrm{ManNH}_{2} ; \mathbf{2 c}\right)$ were used as substrates (Table 1, entries 1-3). Fortunately, when the equivalents of 1 was increased to two or more, the diazotransfer reaction on $\mathrm{GalNH}_{2}(\mathbf{2 a})$ progressed with complete consumption of the amine substrate, indicated by a blue-to-green colour change, and confirmed by TLC analysis (Table 1, Table S1). Similar results were also observed for $\mathrm{GlcNH}_{2}$ (2b) and $\mathrm{ManNH}_{2}$ (2c) substrates. Remarkably, full conversion was also indicated by a blue-to-green colour change when neomycin was used as a substrate, indicating that the hexa-azido product could be formed in 5 min when two or more equivalents of 1 per amine and $1.25 \mathrm{~mol}_{\%} \mathrm{CuSO}_{4}$ were used in the diazotransfer reaction (Table 1, entry 4 ).

Table 1. Optimization of diazotransfer reaction using 1. $^{\text {[a] }}$

\begin{tabular}{|c|c|c|c|c|}
\hline Entry & Substrate & Product & Equiv. $1^{[b]}$ & $\begin{array}{l}\text { Reaction } \\
\text { completed in } 5 \\
\min ^{[c]}\end{array}$ \\
\hline \multirow[t]{3}{*}{1} & \multirow{3}{*}{$\underbrace{}_{2 \mathrm{a} \mathrm{NH}} \cdot \mathrm{HCl}$} & \multirow{3}{*}{$\underbrace{\int_{3}^{\mathrm{OH}}}_{3 \mathrm{a} \mathrm{N}} \mathrm{OH}$} & 1 & No \\
\hline & & & 2 & Yes \\
\hline & & & 5 & Yes \\
\hline \multirow[t]{3}{*}{2} & \multirow{3}{*}{$\underbrace{}_{\mathbf{2 b ~ N H}} \cdot \int_{\mathrm{HCl}}^{\mathrm{OH}}$} & \multirow{3}{*}{$\underbrace{\mathrm{OH}}_{3 \mathbf{b} \mathrm{N}_{3}} \mathrm{OH}$} & 1 & No \\
\hline & & & 2 & Yes \\
\hline & & & 5 & Yes \\
\hline \multirow[t]{3}{*}{3} & \multirow{3}{*}{$\underbrace{\mathrm{HO}}_{2 c}=\mathrm{NH}_{2}^{\mathrm{NH}_{2}} \cdot \mathrm{HCl}$} & \multirow{3}{*}{$\underbrace{\mathrm{HO}-\mathrm{N}_{3}}_{3 c} \mathrm{OH}$} & 1 & No \\
\hline & & & 2 & Yes \\
\hline & & & 5 & Yes \\
\hline \multirow[t]{2}{*}{$4^{[\mathrm{b}]}$} & \multirow{2}{*}{ (NO) } & \multirow{2}{*}{ HO } & 2 & Yes \\
\hline & & & 4 & Yes \\
\hline
\end{tabular}

[a] Substrate was dissolved in a solution of 1 in 1:1 MTBE/DMF and to the mixture was added an aqueous solution of potassium bicarbonate (4 equiv.) and copper (II) sulfate pentahydrate (1.25 mol\%). [b] Equiv. of 1 per amine in substrate. [c] Reaction completion determined after 5 min by colour change from blue to green and TLC analysis. 
With these promising results in hand, we increased the scale of the diazotransfer reaction on common hexosamine substrates to isolate azido sugars, and investigated the substrate scope of our optimized protocol. After a 5 min diazotransfer reaction using 2 equivalents of $\mathrm{FSO}_{2} \mathrm{~N}_{3}(1)$ with $1.25 \mathrm{~mol}_{\%} \mathrm{CuSO}_{4}$, followed by global acetylation, isolated yields were quantitative using $\mathrm{GalNH}_{2}$, $\mathrm{GlcNH}_{2}$ and $\mathrm{ManNH}_{2}$ substrates (Table 2, products 4a-c). Quantitative isolated yields for diazotransfer followed by acetylation were reproducible on mmol scale using $\mathrm{GalNH}_{2}$ as the amine substrate. Diazotransfer with 1 to form the same acetylated azido sugars proceeded in a fraction of the time compared to other reported diazotransfer reagents. ${ }^{[7-8]}$ Furthermore, the preparation of 1 is safer than the alternative diazotizing agents $\left(\mathrm{TfN}_{3}\right.$ and $\left.\mathrm{Im}-\mathrm{SO}_{2} \mathrm{~N}_{3}\right)$.

Table 2. Synthesis of azido sugars from primary amines using 1. ${ }^{[a]}$

\begin{tabular}{|c|c|c|c|}
\hline Entry & Substrate & Product & Yield (\%) \\
\hline $1^{[\mathrm{b}]}$ & $\underbrace{\mathrm{OH}}_{2 \mathrm{a} \mathrm{NH}_{2} \cdot \mathrm{HCl}}$ & $\underbrace{A c O}_{4 a} N_{3}^{O A c}$ & Quant. \\
\hline $2^{[\mathrm{b}]}$ & 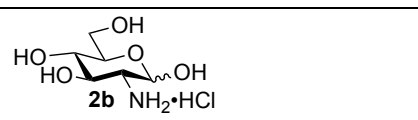 & 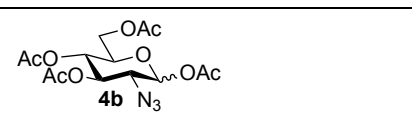 & Quant. \\
\hline $3^{[\mathrm{b}]}$ & ${ }_{2 c}^{\mathrm{HO}} \mathrm{NH}_{2}^{\mathrm{NO}} \cdot \mathrm{HCl}$ & ${ }_{4 c}^{A C O}=\sum_{A c O}^{N_{3}}$ & Quant. \\
\hline 4 & 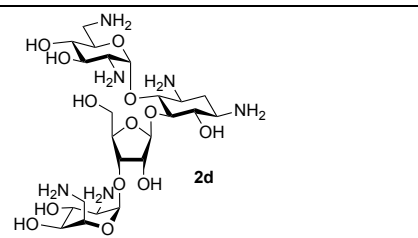 & 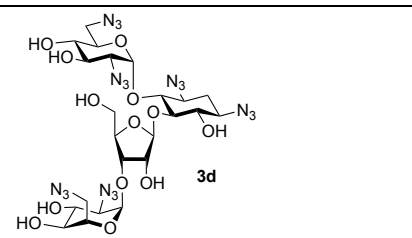 & 83 \\
\hline 5 & 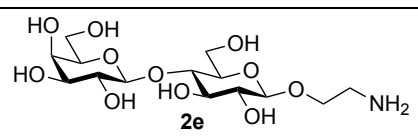 & $\underbrace{\mathrm{HO}}_{\mathrm{OH}} \underbrace{\mathrm{OOH}}_{3 \mathrm{O}} \underbrace{\mathrm{OOH}}_{\mathrm{OH}} \underbrace{\sum_{\mathrm{O}}^{\mathrm{O}}}_{\mathrm{N}_{3}}$ & 87 \\
\hline
\end{tabular}

[a] Substrate was dissolved in a solution of 1 in 1:1 MTBE/DMF and to the mixture was added an aqueous solution of potassium bicarbonate (4 equiv.) and copper (II) sulfate pentahydrate $(1.25 \mathrm{~mol} \%$ ). [b] After workup, azido sugars were acetylated with acetic anhydride, pyridine and DMAP.

High isolated yields (83\%) were also obtained after a 5 min diazotransfer reaction on neomycin, giving hexa-azido product $\mathbf{3 d}$. Furthermore, treatment of aminoethyl-lactoside derivative $\mathbf{2 e}$, which contains a linker at the reducing end functionalized with a primary amine, with 1 afforded azidoethyl-lactoside $3 \mathrm{e}$ (Table 2) in a $87 \%$ yield. Additionally, we sought to determine whether 1 could be stored prior to use in a diazotransfer reaction. Thus, $\mathrm{FSO}_{2} \mathrm{~N}_{3}$ (1) was stored at room temperature in a solution of MTBE/DMF and was subsequently tested in diazotransfer reaction with $\mathrm{GalNH}_{2}(\mathbf{2 a})$. It was found that 1 could be stored for two days without a noticeable loss in 
yield of the acetylated GalN $_{3}$ product (4a), however a modest decrease in yield to $77 \%$ was observed after 7 days of storage prior to use (Table S2).

To demonstrate the utility of this transformation for glycoside synthesis, the optimized diazotransfer protocol was used for the preparation of common glycosyl trichloroacetimidate donors with non-participating C2-azido protecting groups (Scheme 2). Both $\mathrm{GlcNH}_{2}$ and $\mathrm{GaINH}_{2}$ underwent diazotransfer reactions with 1 to afford $\mathbf{3 a}$ and $\mathbf{3 b}$, respectively, which could be globally acetylated furnishing $\mathbf{4 a}$ and $\mathbf{4 b}$ (Scheme 2A). The 2-azido-2-deoxy-D-galactopyranoside (3a) was also protected with a benzylidene acetal, and after acetylation the orthogonally protected galactoside 4d was obtained (Scheme 2B). The globally protected sugars were selectively deprotected at the anomeric position with $\mathrm{NH}_{3}$ in methanol yielding $\mathbf{5 a - c}$, which were readily converted to the trichloroacetimidate donors $\mathbf{6 a}-\mathbf{c}$. Both $\mathrm{GaIN}_{3}$ and $\mathrm{GlcN}_{3}$ donors $\mathbf{6 a}$ and $\mathbf{6 b}$ were prepared in good overall yield (>70\%), with only one column purification after formation of the imidate products (Scheme 2A). When a benzylidene acetal was employed as a protecting group (Scheme 2B), an additional purification step was needed after preparation of $\mathbf{4 d}$. The use of $\mathrm{FSO}_{2} \mathrm{~N}_{3}$ (1) represents a significant improvement in the yield, safety, and time required to afford a similar trichloroacetimidate galactosyl donors through azidonitration or other diazotransfer protocols. ${ }^{[13]}$

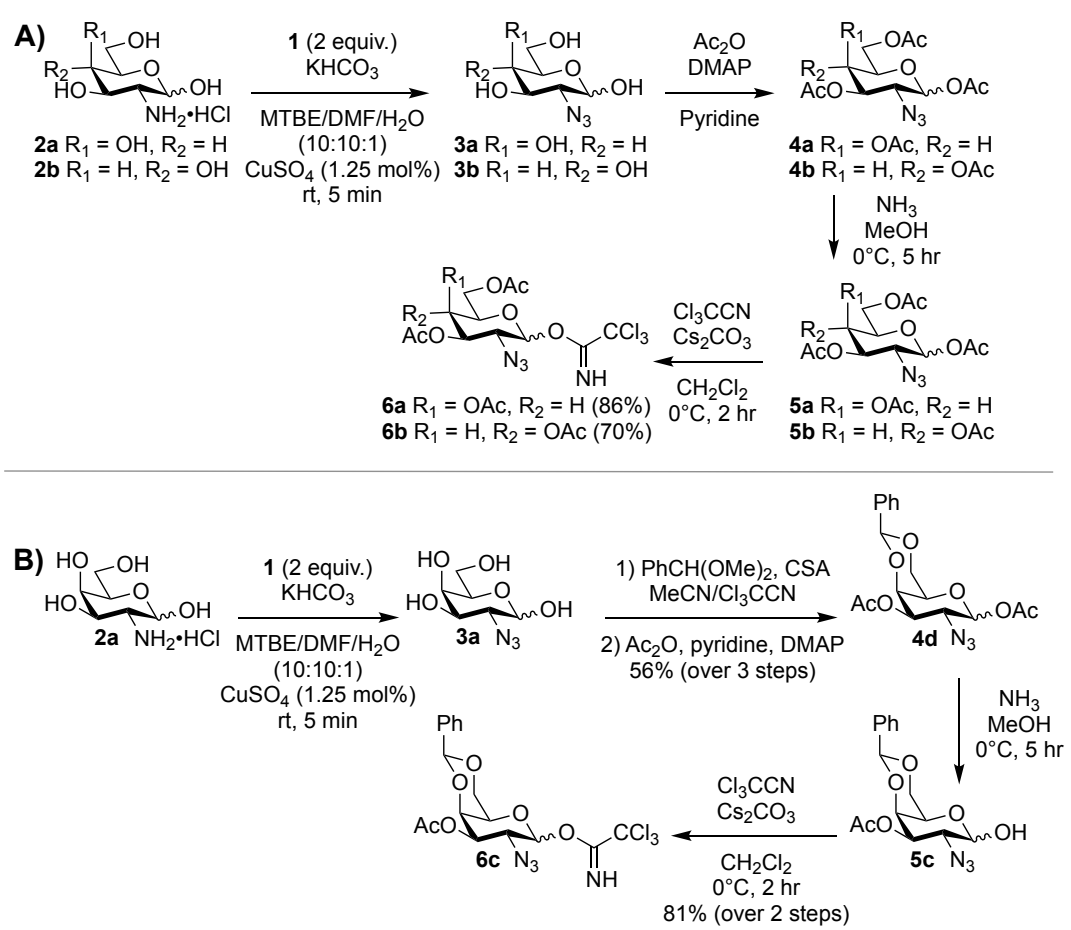

Scheme 2. Application of diazotransfer reaction using 1 for the synthesis of orthogonally protected glycosyl trichloroacetimidate donors. A) Synthesis of peracetylated 2-azidoglycosyl trichloroacetimidate donors 6ab. B) Synthesis of benzylidene acetal-protected 2-azidogalactosyl trichloroacetimidate donor $\mathbf{6 c}$. 


\section{Conclusion}

In summary, we have developed a safer and faster method to synthesize a series of azidecontaining carbohydrates using fluorosulfuryl azide as an efficient diazotransfer reagent. The novel application of 1 for diazotransfer reactions on amino sugars represents a highly reliable and robust route to prepare orthogonally protected azide-containing carbohydrate building blocks compared to previously reported diazotransfer or azidonitration strategies. We demonstrate that this methodology is highly compatible with different 2-amino-2-deoxysugars, carbohydrate derivatives containing an amine-functionalized linker, and aminoglycosides displaying multiple amines, to afford azido sugar analogues in high isolated yields. This optimized method will expand access to the important azide functionality and non-participating C-2 protecting group on carbohydrates, thus allowing for more efficient stereoselective synthesis of 1,2-cis glycosidic linkages.

\section{Acknowledgements}

We acknowledge the National Sciences and Engineering Research Council of Canada (NSERC), the Canada Foundation for Innovation (CFI) and the Banting Research Foundation for funding supporting this work. J.M.K. acknowledges NSERC for a CGS-M scholarship and the Province of Ontario for an OGS award.

Keywords: diazotransfer • azido sugar • azides $\bullet$ carbohydrate chemistry $\bullet$ chemical glycosylation

\section{Works Cited}

[1] a) H. S. G. Beckmann, V. Wittmann, in Organic Azides, 2009, pp. 469-490; b) A. G. Volbeda, G. A. van der Marel, J. D. C. Codée, in Protecting Groups, 2019, pp. 1-27; c) C. Li, L.-X. Wang, Chem. Rev. 2018, 118, 8359-8413; d) A. K. Agrahari, P. Bose, M. K. Jaiswal, S. Rajkhowa, A. S. Singh, S. Hotha, N. Mishra, V. K. Tiwari, Chem. Rev. 2021, 121, 7638-7956; e) R. Sangwan, A. Khanam, P. K. Mandal, Eur. J. Org. Chem. 2020, 2020, 5949-5977; f) K. K. Palaniappan, C. R. Bertozzi, Chem. Rev. 2016, 116, 14277-14306.

[2] a) R. A. Dwek, Chem. Rev. 1996, 96, 683-720; b) H. Herzner, T. Reipen, M. Schultz, H. Kunz, Chem. Rev. 2000, 100, 4495-4538; c) Essentials of Glycobiology, 3rd Edition, Cold Spring Harbor Laboratory Press, Cold Spring Harbor, NY, 2017; d) R. Rai, I. McAlexander, C.-W. T. Chang, Org. Prep. Proced. 
Int. 2005, 37, 337-375; e) A. Varki, Glycobiology 1993, 3, 97-130; f) S. Hakomori, Annu. Rev. Immunol. 1984, 2, 103-126; g) N. Sharon, Biochem. Soc. Trans. 2008, 36, 1457-1460; h) T. Feizi, R. A. Childs, Trends Biochem. Sci. 1985, 10 , 24-29; i) T. Feizi, Trends Biochem. Sci. 1981, 6, 333-335.

[3] a) A. F. G. Bongat, A. V. Demchenko, Carbohydr. Res. 2007, 342, 374-406; b) H. Paulsen, Angew. Chem. Int. Ed. 1982, 21, 155-173; c) S. S. Nigudkar, A. V. Demchenko, Chem. Sci. 2015, 6, 2687-2704.

[4] a) H. Paulsen, W. Stenzel, Chem. Ber. 1978, 111, 2334-2347; b) H. Paulsen, W. Stenzel, Chem. Ber. 1978, 111, 2348-2357.

[5] a) R. U. Lemieux, R. M. Ratcliffe, Can. J. Chem. 1979, 57, 1244-1251; b) S. Czernecki, E. Ayadi, D. Randriamandimby, J. Org. Chem. 1994, 59, 8256-8260;

c) S. J. Danishefsky, M. T. Bilodeau, Angew. Chem. Int. Ed. 1996, 35, 13801419; d) T. Linker, Chemistry 2020, 2, 80-92.

[6] P. H. Seeberger, S. Roehrig, P. Schell, Y. Wang, W. J. Christ, Carbohydr. Res. 2000, 328, 61-69.

[7] A. Vasella, C. Witzig, J.-L. Chiara, M. Martin-Lomas, Helv. Chim. Acta 1991, 74, 2073-2077.

[8] a) A. Titz, Z. Radic, O. Schwardt, B. Ernst, Tetrahedron Lett. 2006, 47, 23832385; b) P. T. Nyffeler, C.-H. Liang, K. M. Koeller, C.-H. Wong, J. Am. Chem. Soc. 2002, 124, 10773-10778; c) H. Ye, R. Liu, D. Li, Y. Liu, H. Yuan, W. Guo, L. Zhou, X. Cao, H. Tian, J. Shen, P. G. Wang, Org. Lett. 2013, 15, 18-21; d) P. B. Alper, S.-C. Hung, C.-H. Wong, Tetrahedron Lett. 1996, 37, 6029-6032; e) E. D. Goddard-Borger, R. V. Stick, Org. Lett. 2007, 9, 3797-3800; f) R.-B. Yan, F. Yang, Y. Wu, L.-H. Zhang, X.-S. Ye, Tetrahedron Lett. 2005, 46, 8993-8995; g) S. Schoffelen, M. B. van Eldijk, B. Rooijakkers, R. Raijmakers, A. J. R. Heck, J. C. M. van Hest, Chem. Sci. 2011, 2, 701-705.

[9] C. J. Cavender, V. J. Shiner, J. Org. Chem. 1972, 37, 3567-3569.

[10] E. D. Goddard-Borger, R. V. Stick, Org. Lett. 2011, 13, 2514-2514.

[11] G. Meng, T. Guo, T. Ma, J. Zhang, Y. Shen, K. B. Sharpless, J. Dong, Nature 2019, 574, 86-89.

[12] T. Guo, G. Meng, X. Zhan, Q. Yang, T. Ma, L. Xu, K. B. Sharpless, J. Dong, Angew. Chem. Int. Ed. 2018, 57, 2605-2610.

[13] a) G. Grundler, R. R. Schmidt, Liebigs Ann. Chem. 1984, 1984, 1826-1847; b) E. Bedini, L. Cirillo, M. Parrilli, Carbohydr. Res. 2012, 349, 24-32. 